\title{
Evaluation of Eimeria oocyst whole antigen vaccine and the enhancive effect of probiotic on broilers
}

\author{
Nasr M. Elbahy ${ }^{2}$, Reda E. Khalafalla ${ }^{1 *}$, Ahmed O. Elkhatam ${ }^{2}$, Mahmoud AbouLaila ${ }^{2}$ \\ ${ }^{I}$ Department of Parasitology, Fac. Vet. Med., Kafrelsheikh University, Kafr El-Sheikh, Egypt \\ ${ }^{2}$ Department of Parasitology, Faculty of Veterinary Medicine, Sadat City University, Minufiya, Egypt \\ *Corresponding author E-mail: redabast@hotmail.de
}

Copyright () 2014 Reda E. Khalafalla e al. This is an open access article distributed under the Creative Commons Attribution License, which permits unrestricted use, distribution, and reproduction in any medium, provided the original work is properly cited.

\begin{abstract}
Chicken coccidiosis has great economic impacts on poultry industry. The present study was conducted to evaluate the effect of feeding probiotic (Biopellt-s) as well as Eimeria oocyst whole antigen vaccine by subcutaneous and intraperitoneal routes on body weight gain, lesion score, oocyst output, and phagocytic activity. One-day old broiler chicks were divided into seven equal groups (50 chicks each); control negative group (C-Ve), probiotic fed group (PRO), subcutaneous vaccinated group (S/C), intraperitoneal vaccinated group (I/P), S/C plus probiotic (S/C Pro), I/P plus probiotic (I/P Pro), and positive control group $(\mathrm{C}+\mathrm{Ve})$. At four weeks, chicks challenged with 12.000 sporulated oocysts. Vaccination decreases the lesion score and the oocyst output in S/C group (9000 oocyst/gram) and in I/P group (12500 oocyst/gram) compared with positive control (43500 oocyst/gram). S/C was more effective than I/P for controlling the infection.

Probiotic supplementation resulted in significant increase in body weight gain (1323 gm) compared with positive control (1026.667 gm) and phagocytic activity (22.83\%) compared with positive control (16.08\%) while the vaccination was more effective than probiotic alone. S/C vaccination with probiotic was more effective than with $\mathrm{I} / \mathrm{P}$ vaccination. Therefore, this study recommends the use of S/c or I/P vaccination regimens for coccidiosis.
\end{abstract}

Keywords: Chicken Coccidiosis, Eimeria Oocyst Whole Antigen Vaccine, Probiotic.

\section{Introduction}

Avian coccidiosis is a disease that is universally found wherever chickens are raised and causes high economic losses to poultry industry [1]. Coccidiosis is caused by Eimeria spp. such as E. tenella, E. necatrix, E. brunetti, E. maxima, E. mitis, and E. acervulina [2].Coccidiosis is a self-limiting infectious disease of the digestive tract. In the heavy infection, it induces a sever necrosis to the intestinal mucosa, hemorrhages, and unlimitedly death [3], [4].

Anticoccidial drugs have been reasonably effective on the preventing serious outbreaks of coccidiosis among birds reared for broiler market. However, the life of most of these drugs is limited due to the emergence of resistant strains of Eimeria. Therefore, a pressing need for an alternative method of control is required.

Vaccination is one of the methods for control of Coccidiosis. It starts by the use of either life virulent as Cocci vac B or life attenuated as Paracox vaccines that contain the important pathogenic species of Eimeria [5]. Despite the development of good immune response able to protect the birds and its effect on decreasing the resistant to anticoccidials after mixing with the resistant strains, live vaccines induce lesions that may adversely affect the health of the birds and perhaps increase their susceptibility to secondary infection with pathogens, such as Clostridium perfringes causing necrotic enteritis [5]. Therefore, vaccination with dead whole oocyst antigen from different species of Eimeria overcame this problem. Vaccination with $0.2 \mathrm{mg}$ by Subcutaneous and intraperitoneal routes successfully protected chicks from the challenge infection with Eimeria infection [6], [7].

Probiotic is a preparation or viable micro-organisms, which is consumed by humans or other animals with the aim of inducing beneficial effects by qualitatively or quantitatively influencing their gut microflora and/or modifying their immune status [8]. Probiotic and vaccination increase immunity of chickens against coccidiosis [9], [10]. The aim of the 
present study was to evaluate the effect of Subcutaneous and intraperitoneal whole antigen vaccine against broilers coccidiosis and enhancive effect of probiotic.

\section{Materials and methods}

\subsection{Animals}

Three-hundred Hubbard one-day old chicks were obtained from a local commercial broiler hatchery. The chickens were subjected to the following vaccination schedule; Hitchner B1at $6^{\text {th }}$ day, IBD intermediate strain at 13 and 20 days, Lasota at 17, 27, and 37 days of age. All vaccines were given via drinking water. They were reared under hygienic condition and fed on balanced ration and water ad libitum (free from antibacterial agent according to Harrison and Harrison [11]. The prepared ration (not less than $21 \%$ crude protein; not less than $2.8 \%$ crude fat and not more than $3.1 \%$ crude fiber).

\subsection{Probiotic (biopellt-s) $R$}

Probiotic was used at a rate of $2 \mathrm{gm} / 1$ Littre water.

\subsection{Eimeria strains}

A field strain of sporulated Eimeria oocysts were maintained at the Deptartment of Parasitology, Faculty of Veterinary Medicine, and University of Sadat City by passages in chickens. Mixture of the oocysts consists of E. tenella $30 \%, E$. maxima $20 \%$, E. acervulina $20 \%$, E. necatrix $15 \%$, and E. mitis $15 \%$.

\subsection{Antigen preparation}

Whole Eimeria oocyst antigen was prepared and the content was quantified according to the technique of Saotara Oz et al. [12].

\subsection{Experimental design}

The chicks were grouped (fifty chicks per group) according to the dose of a mixture of oocysts given to each chick, also the method of inoculation and type of probiotic used. Mixture of the oocysts consists of (E. tenella 30\%, E. maxima $20 \%$, E. acervulina $20 \%$, E. necatrix $15 \%$, and E. mitis $15 \%$ ). groups were divided as follows:-Group (1) was injected with 0.2 $\mathrm{mg}$ whole oocysts antigen/chick I/p at the first 5 days of age Group (2) was injected with 0.2 mg whole oocysts antigen/chick S/C at the first 5 days of age Group (3) was given Bio pellet-s $2 \mathrm{gm} / \mathrm{L}$ drinking water from the first day till the end of experiment and injected with $0.2 \mathrm{mg}$ whole oocysts antigen/chick $\mathrm{I} / \mathrm{p}$ at the first 5 days of age. Group (4) was given Bio pellet-s $2 \mathrm{gm} / \mathrm{L}$ drinking water from the first day till the end of experiment and injected with $0.2 \mathrm{mg}$ whole oocysts antigen/chick S/C at the first 5 days of age. After two weeks the four groups were given a booster dose. Group (5) was given Bio pellet-s $2 \mathrm{gm} / \mathrm{L}$ drinking water from the first day till the end of experiment. Group 6 represents positive control group, infected non-immunized and without probiotic. Group 7 was non-infected control negative group (C-ve group). At 4 weeks, groups 1, 2, 3, 4, 5, and 6 were challenged with 12.000 sporulated oocysts/birds orally. Oocyst counting was performed at days 5, 6, 7, 8 post challenge. Five chicks were sacrificed one week post-vaccination as well as one week post-challenge for serum collection and recording lesion score.

\subsection{Examination of the birds}

\subsubsection{Body weight gain}

Checks in all groups were weighted every 2 weeks till week 6 to determine the effect of probiotic on body weight gain in comparison with non-probiotic group. Body weight gain was expressed in grams.

\subsubsection{Clinical examination}

The clinical sings as depression, diarrhea, and loss of appetite were recorded during experimental period. Post mortem examination performed to intestine at one week post-challenge and lesion score determined according to Table 1. 
Table 1: Lesion Score

\begin{tabular}{|c|c|}
\hline Grade & Remarks \\
\hline Grade 1 & $\begin{array}{l}\text { - Light redness of the intestinal wall. } \\
\text { - Mild thickening of the intestinal wall. } \\
\cdot 1-3 \text { focal lesions in } 3 \mathrm{~cm} \text {. of the intestinal wall. }\end{array}$ \\
\hline Grade 2 & $\begin{array}{l}\text { - Moderate redness of the intestinal wall. } \\
\text { - Moderate thickening of the intestinal wall. } \\
\text { - 3-6 focal lesions in } 3 \mathrm{~cm} \text {. of the intestinal wall. } \\
\text { - Ballooning in the caecum. }\end{array}$ \\
\hline Grade 3 & $\begin{array}{l}\text { - Severe congestion of the intestinal wall. } \\
\text { - Increase the thickening of the intestinal wall. } \\
\text { - Ballooning in the caecum and presence of "bloody cecal core". }\end{array}$ \\
\hline
\end{tabular}

\subsubsection{Oocyst count}

Oocyst count was performed using the McMaster counting technique according to the method described by Long and Joyner [13].

\subsection{Electrophoretic pattern of serum proteins}

Sodium dodecyl-sulphate-polyacrylamide gel electrophoresis (SDS-PAGE) of serum sample was carried out according to the procedure of Laemmli [14]. The molecular weight was determined using calibration curve according to Laemmli [14]. The gel was scaned using "Bio-Rad Model GS-670, Imaging Densitometer" at wave length $605 \mathrm{~nm}$. Different serum fractions were quantified with a polar planimeter. The area of each profile fraction was calculated as a percentage of the whole. Comparisons were then made among profiles on the basis of these percentages for correspondingly fractions.

\subsection{Phagocytic activity of neutrophils}

Phagocytic activity of polymorph nuclear cells using Candida albicans was performed according to the method described by Wilkinson [15]. Briefly, aliquots of $100 \mu \mathrm{l}$ of fetal calf serum, heat killed Candida albicans $(5 \mathrm{x} 106 / \mathrm{ml})$ and blood were mixed in a plastic tube. The tube was mixed and incubated at $37 \mathrm{C}$ for 30 minutes, after which it was centrifuged at $1000 \mathrm{rpm}$ for 5 minutes. The supernatant was discarded leaving a droplet into which the sediment was resuspended. Smears were prepared from the deposit, dried in the air, fixed with methyl alcohol and stained with Giemsa stain. One hundred neutrophils were examined and the number of neutrophils ingesting Candida albicans was counted and expressed as percentage.

\subsection{Statistical analysis}

Data obtained from the results of the present study were subjected to analysis by student's-t-test using SPSS 11 . *: Significant at $\mathrm{P}<0.05$, **: Highly significant at $\mathrm{P}<0.01$, and $* * *$ : Very highly significant at $\mathrm{P}<0.001$

\section{Results}

\subsection{Body weight gain}

There was significant increase in body weight in vaccinated plus probiotic and probiotic groups compared with the negative control group at the end of the $2^{\text {nd }}$ and $4^{\text {th }}$ weeks (Table 2). There was significant decrease in body weight of positive control group compared to the control negative group at the end of the week $6^{\text {th }}$ (Table 2 ).

\subsection{Clinical examination}

\subsubsection{Clinical signs}

In vaccinated $(\mathrm{G} 1,2)$, vaccinated and supplemented with probiotic $(\mathrm{G} 3,4)$, and supplemented with probiotic(G5) groups, the symptoms were similar to negative control group (G7) but all birds showed depression, in the early days after injection (vaccinated) then returns to the normal condition. After challenge chicks showed, depression, decrease the activity and slight bloody diarrhea. Infected non-treated group (G6) showed ruffled feathers, decrease of appetite, anorexia, depression, bloody diarrhea and paralysis. Symptoms started from $5^{\text {th }}$ day post-challenge with maximum 
strength at $7^{\text {th }}$ day post-challenge and then regression and disappearance of bloody diarrhea till the end of the experiment.

\subsubsection{Lesion score}

Group vaccinated S/C (G2) showed slight congestion of all intestinal mucosa with mild thickening of the intestinal wall (Grade 1). Group vaccinated S/C plus probiotic (G4) showed slight congestion of all intestinal mucosa and the ceci were nearly normal from $5^{\text {th }}$ day post-challenge till the end of the experiment (Grade 1). Group vaccinated I/P (G1) showed congestion and hemorrhagic patches of the intestinal mucosa and the ceca were ballooned (Grade 2$)$ from $5^{\text {th }}$ to $7^{\text {th }}$ days post-challenge. Group vaccinated I/P plus probiotic (G3) showed gradual increase of congestion of all the intestinal mucosa and the ceca were slightly ballooned (Grade 2) from $5^{\text {th }}$ to $7^{\text {th }}$ days post-challenge. Positive control (G6) showed increase of congestion of all intestinal mucosa with presence of cecal core and increase the thickening of intestinal mucosa (Grade 3 ) from $7^{\text {th }}$ day post-challenge till the end of the experiment.

Table 2: Effect of Vaccination and Probiotic on Body Weight Gain (Gm.) (Mean \pm S.E) In Different Groups

\begin{tabular}{lllc}
\multicolumn{2}{c}{ Table 2: Effect of Vaccination and Probiotic on Body Weight Gain (Gm.) (Mean \pm S.E) In Different Groups } \\
\hline Groups & 2 weeks & 4 weeks & 6 weeks \\
\hline I/P & $451.33 \pm 2.40$ & $940.25 \pm 5.62$ & $1323.33 \pm 14.529$ \\
S/C & $460.00 \pm 2.89$ & $933.45 \pm 3.27$ & $1310.00 \pm 20.82$ \\
I/P Pro & $526.88 \pm 3.45^{*}$ & $1154 \pm 3.06^{* * *}$ & $1426.67 \pm 14.529$ \\
S/C Pro & $527.83 \pm 4.05^{*}$ & $1010.33 \pm 5.94^{* *}$ & $1396.67 \pm 8.819$ \\
Pro & $622.00 \pm 4.16^{* *}$ & $1066.81 \pm 8.93^{* *}$ & $1323.00 \pm 14.53$ \\
C + Ve & $349.9 \pm 8.72$ & $707.2 \pm 10.32$ & $1026.667 \pm 14.529^{* *}$ \\
C-Ve & $460.48 \pm 1.08$ & $930.14 \pm 5.77$ & $1350.00 \pm 28.87$ \\
\hline
\end{tabular}

\subsection{Oocyst count}

The oocyst count was significantly decreased after $0.2 \mathrm{mg}$ of Eimeria whole antigen immunization, by S/C or I/P routes with or without probiotic, as well as probiotic treatment than the oocyst count in the control positive group. In the S/C group, it reached the peak of 9000 oocyst/gram. In the I/P group, the peak oocyst count was 12500 oocyst/gram. In the S/C Pro group, the peak oocyst count was 9000 oocyst/gram. In the I/P Pro group, the peak oocyst count was 12500 oocyst /gram. In the Pro group, the peak oocyst count was 19000 oocyst /gram. While in the control positive group, the peak oocyst count was 43500 oocyst /gram compared with less than 100 oocysts /gram for the negative control group that was considered as zero oocyst per gram.

\subsection{Immunological effect of vaccination and/ or probiotic}

\subsubsection{Protein gel electrophoresis}

There was significant increase in serum alpha, beta and gamma globulins of S/C, S/C Pro, I/P Pro, and Pro groups one week post vaccination while there was significant increase in serum gamma globulins of I/P group compared to the control group (Table 3 and Fig. 1 A). One week post challenge, there was significant increase in gamma globulins in $\mathrm{S} / \mathrm{C}$ pro while there is insignificant change in beta and alpha compared with control group (Table 4 and Fig. 1B). In the I/P Pro group, there was significant increase in gamma while insignificant increase in beta and alpha. In Pro group, there was significant increase in gamma and alpha with insignificant change in beta compared to control negative group. In the $\mathrm{S} / \mathrm{c}$ and $\mathrm{I} / \mathrm{P}$ groups, there were significant increase in gamma globulins (Table 4). There was insignificant increase in serum gamma, beta, and alpha globulins of control positive group compared to negative control group.

Table 3: Serum Electrophoretic Pattern One Week Post Vaccination in Different Groups

\begin{tabular}{|c|c|c|c|c|c|c|c|}
\hline Group & $\mathrm{C}-\mathrm{Ve}$ & $\mathrm{C}+\mathrm{Ve}$ & $\mathrm{S} / \mathrm{C}$ & $\mathrm{I} / \mathrm{P}$ & S/C Pro & I/P Pro & Pro \\
\hline Gamma & $0.84 \pm 0.01$ & $0.87 \pm 0.03$ & $1.07 \pm 0.01^{*}$ & $1.04 \pm 0.05^{*}$ & $1.42 \pm 0.05^{* * *}$ & $1.36 \pm 0.07^{*}$ & $1.20 \pm 0.07^{*}$ \\
\hline Beta & $0.65 \pm 0.01$ & $0.66 \pm 0.01$ & $0.78 \pm 0.05^{*}$ & $0.71 \pm 0.05$ & $0.85 \pm 0.04^{*}$ & $0.82 \pm 0.01^{* *}$ & $0.81 \pm 0.01^{*}$ \\
\hline Alpha & $0.43 \pm 0.03$ & $0.44 \pm 0.02$ & $0.60 \pm 0.05^{*}$ & $0.50 \pm 0.03$ & $0.60 \pm 0.03^{*}$ & $0.59 \pm 0.09^{*}$ & $0.63 \pm 0.05^{*}$ \\
\hline
\end{tabular}

Table 4: Serum Electrophoretic Pattern One Week Post Challenge in Different Groups

\begin{tabular}{|c|c|c|c|c|c|c|c|}
\hline Group & $\mathrm{C}-\mathrm{Ve}$ & $\mathrm{C}+\mathrm{Ve}$ & $\mathrm{S} / \mathrm{C}$ & $\mathrm{I} / \mathrm{P}$ & S/C Pro & I/P Pro & Pro \\
\hline Gamma & $0.76 \pm 0.01$ & $0.74 \pm 0.01$ & $0.88 \pm .01^{*}$ & $0.89 \pm 0.06^{*}$ & $0.93 \pm .01^{* * *}$ & $0.91 \pm 0.06^{*}$ & $0.90 \pm 0.05^{*}$ \\
\hline Beta & $0.58 \pm 0.02$ & $0.52 \pm 0.01$ & $0.6 \pm 0.01$ & $0.62 \pm 0.01$ & $0.64 \pm 0.03$ & $0.64 \pm 0.02$ & $0.57 \pm 0.06$ \\
\hline Alpha & $0.45 \pm 0.01$ & $0.37 \pm 0.01^{*}$ & $0.45 \pm 0.01$ & $0.52 \pm 0.03$ & $0.47 \pm 0.04$ & $0.47 \pm 0.04$ & $0.57 \pm 0.02$ \\
\hline
\end{tabular}



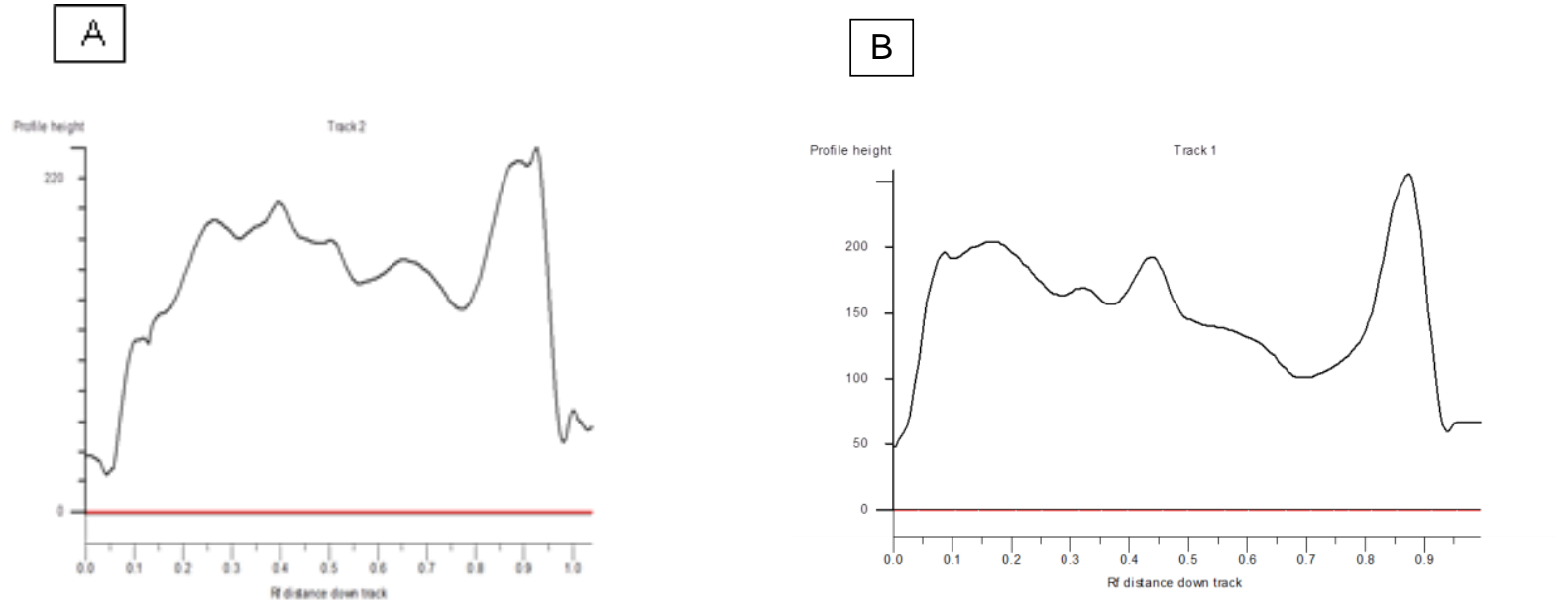

Fig. 1: Serum Electrophoretic Pattern in Broilers in Control Group (A) and in S/C Pro Group (B).

\subsubsection{Phagocytic activity}

Table 5 showed a significant increase in phagocytic activity of all the treated groups (S/C, I/P, S/C Pro, I/P Pro and Pro) compared with the control positive group at 2, 4, and 6 weeks.

Table 5: Phagocytic Activity (Mean \pm S.E) In Different Groups

\begin{tabular}{llll}
\hline Groups & 2 weeks & 4 weeks & 6 weeks \\
\hline I/P & $21.16 \pm 0.68^{*}$ & $22.58 \pm 0.41^{* *}$ & $23.687 \pm 0.258^{* * *}$ \\
S/C & $21.56 \pm 1.08^{* *}$ & $22.43 \pm 0.28^{* *}$ & $24.33 \pm 0.27^{* *}$ \\
I/P Pro & $22.36 \pm 0.80^{* *}$ & $23.95 \pm 0.50^{* *}$ & $23.74 \pm 0.32^{* *}$ \\
S/C Pro & $22.53 \pm 0.40^{* *}$ & $23.85 \pm 0.41^{* *}$ & $24.00 \pm 0.10^{*}$ \\
Pro & $19.07 \pm 0.39^{*}$ & $21.83 \pm 0.54^{*}$ & $22.83 \pm 0.60^{*}$ \\
C+Ve & $15 \pm 0.45$ & $16 \pm .44$ & $16.08 \pm 0.469$ \\
C- ve & $16.36 \pm 0.41$ & $17.3 \pm 0.43$ & $18.40 \pm 0.39$ \\
\hline
\end{tabular}

\section{Discussion}

This study attempts to evaluate the use of vaccines and probiotic (Bacillus subtilis) on coccidiosis. Chickens vaccinated with whole oocysts antigen S/C or I/P showed slight clinical manifestation of illness all over the experimental period even after challenge, decrease lesion score, and oocyst output. This may be due to the sensitization of the antibody mechanism which decrease the propagation of oocyst and prevent the sporozoite from attacking the epithelial lining of the intestine. These results agreed with previously reported work of El-Bahy et al., [7] who mentioned increase of antibodies against sporozoites, [16], [17], and [18]. While vaccinated groups are similar to S/C Pro and I/P Pro groups and higher than Probiotic group. This may be due to probiotic increase protein synthesis especially alpha, beta, and gamma globulins which produce a healthy media.

Probiotic organisms in the gut can produce products such as free radicals, hydrogen peroxide, short-chain fatty acids and other oxygen metabolites [19] that could conceivably harm motile zoites directly and/or affect intracellular stages thereby reducing their numbers. Probiotics modify receptors on enterocytes [19]. This could either impair or destroy sporozoites and/or merozoites from penetrating an enterocyte. The results are in agreement with Lee [20]. On the other hand, Pro group had better clinical signs, lesion score, and oocyst output than the control positive group and this agrees with Mian et al. [21] and Ruiz \& Tamasaukas [22]. Therefore, vaccination is better than probiotic alone in controlling coccidiosis. Furthermore, gel electrophoresis showed that vaccination and supplementation with probiotic induce high immunity. These results agree with Yun et al., [23] who found that probiotics increase the concentration of serum immunoglobulins.

Vaccination has a significant improving effect on phagocytic activity and this in agreement with Awad et al. [24]. The phagocytic activity in vaccinated groups was higher than that in Pro group and vaccinated with Pro groups.

Broiler chicken treated with probiotics showed a significant increase in body weight this due to decrease the effects associated with infection that negatively affect production parameters of these broilers [25].

\section{Conclusion}

In conclusion, this study showed that $\mathrm{S} / \mathrm{C}$ and $\mathrm{I} / \mathrm{P}$ vaccination regimes protected the broiler chickens from effect of coccidia infection and S/C was more effective than I/P. Furthermore, probiotic improved body weight gain, phagocytic 
activity and decrease lesion score and clinical signs. Therefore, this study recommends the use of vaccination regimens alone or with probiotic for protection of broiler chicken against coccidiosis.

\section{References}

[1] B.S. Bhogal, G.A. Miller, A.C. Anderson, E.J. Jesse, S. Strausberg, R. Mc Candliss, and R.L. Strausberg, Vaccination of chickens with recombinant Eimeria tenella antigen alone or in combination with a subclinical exposure induces cross protective immunity against coccidiosis. Progress Clinical and Biological Research, 307 (1988) 131-146.

[2] G.M. Urquhart, J. Amour, J.L. Duncan, A.M. Dunn and K.W. Jennings, Veterinary Parasitology. 2nd ed. Alden press, Great Britain, (1996) pp: 255.

[3] M.K. El-Sayed, The effect of diclazuril and semduramicin as prophylactic or therapeutic treatments $\mathrm{n}$ broilers infected with Eimeria tenella. MVSc. Thesis (Pharmacology), Faculty of Veterinary Medicine, Tanta University (2002).

[4] P.A. Holds Worth, D.P. Conway, M.E. Mekenzie, A.D. Dayton, H.D. Chapman, J.T. Skinner, H.C. Mundt, and R.B. Williams, World Association for the Advancement of Vet. Parasitol., guidelines for evaluating the efficacy of anticoccidial drugs in chickens and turkeys. Vet. Parasitol. 121 (3/4) (2004) 189-212. http://dx.doi.org/10.1016/j.vetpar.2004.03.006.

[5] H.D. Chapman, T.F. Cherry, H.D. Danforth, G. Richards, M.W. Shirley, and Williams R.B., Sustainable coccidiosis control in poultry production: the role of live vaccines. International Journal for Parasitology, 32 (2002) 617-629. http://dx.doi.org/10.1016/S0020-7519 (01)00362-9.

[6] S.S. Khalaf-Allah, A trial to control cecal coccidiosis by immunization of broiler chickens with E. tenella sporozoite antigen. J. Egypt. Vet. Med. Assoc., 62 (2) (2002) 43 -54.

[7] N.M. El-Bahy, A.M. Abul-magd, and R. El Meghanawy, Trails for immunization against coccidiosis in broiler chickens as a control measure. Minufiya Vet. J. 5 (3) (2008) 737-742.

[8] A. Torres-Rodriguez, A.M. Donoghue, D.J. Donaghue, J.T. Barton, G. Tellex and B.M. Hargis, Performance and condemnation rate analysis of commercial turkey flocks treated with a Lactobacillus spp.-based probiotic. Poult. Sci., 86 (2007) $444-446$. http://dx.doi.org/10.1093/ps/86.3.444.

[9] C.F. Crouch, S.J. Anfrews, R.G. Ward and M.J. Francis, Protective efficacy of a live attenuated anti-coccidial vaccine administered to 1 dayold chickens. Avian Pathol. 32 (3) (2003) 297- 304. http://dx.doi.org/10.1080/10307945031000097912.

[10] S.O. Wu, M. Wang, O. Liu, I.J. Zhu, X. Suo, and J.S. Jiang Construction of DNA vaccines and their induced protective immunity against experimental Eimeria tenella infection. Parasitol. Res., 23 (2004) 156-167.

[11] G. J. Harrison and L.R. Harrison, Clinical Avian Medicine and Surgery, WB Saunders company Philadelphia, London. Toronto (1986).

[12] H. Saotara Oz, R.J. Markham, W.J. Bemrick and B.E. Stromberg, Enzyme linked immonosorbant assay and indirect Haemagglutination technique for measurement of antibody responses to E. tenella in experimentally infected chickens. J. Parasitol., 70 (6) (1984) $859-863$. http://dx.doi.org/10.2307/3281630.

[13] P.L. Long and L.P. Joyner, A guide to laboratory techniques used in the study and diagnosis of avian coccidiosis. Folia Vet. Lat. 6, (1976) 201-217

[14] A.R. Laemmli, Clevage of structural proteins during assembly of the head of bacteriophage T4. Nature, 227 (1970) 600-685. http://dx.doi.org/10.1038/227680a0.

[15] P.C. Wilkinson, Techniques in Clinical Immunology. 2nd edition, Black well scientific publication, London (1981).

[16] A.C. Bushell, M.W. Shirley, and J.E. Bushell, The use of an attenuated coccidiosis vaccine in replacement layers. Zootenica International., (5) (1992) 58-62.

[17] S.H. Hegazi, Sorne biological immunological studies on Eimeria steidai of rat bits under the Egyptian environmental condition. Thesis, Ph.D (Parasitological) Faculty of Veterinary Medicine, Cairo University (1988).

[18] H. Tojo and S. Okamoto, Studies on immunity to Eimeria tenella in chickens. Changes in the number of parasites in the caecum and relationship between circulatory antibody and resistance to infection in immune chickens. Japanese Poult. Sci., 8, (3) (1971) 156-160. http://dx.doi.org/10.2141/jpsa.8.156.

[19] L.J. Fooks and G.R. Gibson, Probiotics as modulators of the gut flora. British Journal of Nutrition, 88 (2002) $39-49$. http://dx.doi.org/10.1079/BJN2002628.

[20] S.H. Lee, Use of probiotic formulation in control of Eimeria tenella infection in broiler chickens. Thesis, M.V.Sc., University of Guelph (2008).

[21] M.A. Mian, A. Masood, M. Faqir, U.L. Ahsan, M. Haq and A. Irfan, Experimental Parasitology 128, (2011) 104-110. http://dx.doi.org/10.1016/j.exppara.2011.02.024.

[22] H. Ruiz and R. Tamasaukas, Immunoprotection: an avian coccidiosis in the fowl. Experimental Parasitol., 42 (1) (1995) 129 - 141.

[23] C.H. Yun, H.S. Lillehoj, and E.P. Lillehoj, Intestinal immune response to coccidiosis. Developmental and Comparative Immunology, 24 (2000) 303-324. http://dx.doi.org/10.1016/S0145-305X (99)00080-4.

[24] A.M. Awad, A. F. El-Nahas, and S.S. Abu-Akkada, Evaluation of protective efficacy of anticoccidial vaccine, Coccivac B® in broiler chicks in relation to sequence analysis of ITS1 gene using five Egyptian field strains of Eimeria tenella. Minoufiya Veterinary Journal (2012) 19-45.

[25] H.R. Haghighi, J. Gong, C.L. Gyles, A. Hayes, H. Zhou, B. Sanei, J.R. Chambers and S. Sharif, Probiotics stimulate production of natural antibodies in chickens. Clinical and Vaccine Immunology, 13 (2006) 975-980. http://dx.doi.org/10.1128/CVI.00161-06. 